\title{
Microcystin-LR activates the ERK1/2 kinases and stimulates the proliferation of the monkey kidney-derived cell line Vero-E6
}

\author{
E. Dias ${ }^{\text {a,b,c,* }}$, P. Matos ${ }^{\text {a }}$, P. Pereira ${ }^{b}$, M.C.C. Batoréu $^{c}$, M.J. Silva ${ }^{\text {a }}$, P. Jordan ${ }^{\text {a }}$ \\ ${ }^{a}$ National Institute of Health Dr. Ricardo Jorge, Department of Genetics, Av. Padre Cruz, 1649-016 Lisbon, Portugal \\ ${ }^{\mathrm{b}}$ National Institute of Health Dr. Ricardo Jorge, Department of Environmental Health, Av. Padre Cruz, 1649-016 Lisbon, Portugal \\ ' University of Lisbon, Faculty of Pharmacy, I-Med, Laboratory of Toxicology, Av. Prof. Gama Pinto, 1649-019 Lisbon, Portugal
}

\section{A R T I C L E I N F O}

\section{Article history:}

Received 25 November 2009

Accepted 21 May 2010

Available online $\mathrm{xxxx}$

\section{Keywords:}

Microcystin-LR

Tumour promotion

ERK1/2

ERK1/2

Kidney

\begin{abstract}
A B S T R A C T
Microcystin-LR (MCLR) is a peptide produced by freshwater cyanobacteria that induces severe hepatotoxicity in humans and animals. MCLR is also a potent tumour promoter and it has been proposed that this activity is mediated by the inhibition of protein phosphatases PP1/PP2A, possibly through the activation of proto-oncogenes c-jun, $c$-fos and c-myc. However, the mechanisms underlying MCLR-induced tumour promotion are still largely unknown, particularly in non-liver cells. In previous studies we have demonstrated that micromolar concentrations of MCLR induce cytotoxic effects in the kidney Vero-E6 cell line. The purpose of the present work was to evaluate whether the exposure to subcytotoxic concentrations of MCLR was sufficient to induce the proliferation of Vero-E6 cells. Through BrdU incorporation assay we show that at nanomolar concentrations MCLR stimulates cell cycle progression in Vero-E6 kidney cell line. Moreover, the analysis of mitogen-activated protein kinases p38, JNK and ERK1/2 activity revealed that the proliferative effect of MCLR is associated with the activation of the pro-proliferative ERK1/2 pathway. These results emphasise the importance to confirm in vivo the impact of MCLR on tumour promotion at kidney level.
\end{abstract}

(c) 2010 Elsevier Ltd. All rights reserved.

\section{Introduction}

Microcystins (MC) are secondary metabolites produced by freshwater cyanobacteria that have been associated with severe episodes of human and animal acute hepatotoxicity (Codd et al., 2005; van Apeldoorn et al., 2007). MC are a group of approximately 70 structural variants sharing the common cyclic heptapeptide structure cyclo(-D-Ala ${ }^{1}-\mathrm{L}-\mathrm{X}^{2}-\mathrm{MeAsp}^{3}-\mathrm{L}-\mathrm{Z}^{4}-\mathrm{Adda}^{5}-\mathrm{D}-\mathrm{Glu}^{6}-\mathrm{N}-\mathrm{Mdha}^{7}$ ) where $\mathrm{X}$ and $\mathrm{Z}$ are variable $\mathrm{L}$-amino acids. MeAsp, Mdha and Adda are abbreviations of methylaspartic acid, methyldehydroalanine and 3-amino-9-methoxy-2,6,8-trimethyl-10-phenyldeca-4,6-dienoic acid, respectively (Chen et al., 2006). MCLR (with leucine-Land arginine-R- in the variable positions) is one of the most frequent and acutely toxic variant (Funari and Testai, 2008). MC is produced by several cyanobacteria species from distinct genera such as Microcystis, Anabaena, Planktothrix (van Apeldoorn et al., 2007). Under favourable environmental conditions, particularly in eutrophic water resources, those organisms proliferate massively forming dense biomass concentrations (bloom) that, during the

\footnotetext{
* Corresponding author at: National Institute of Health Dr. Ricardo Jorge, Department of Genetics, Av. Padre Cruz, 1649-016 Lisbon, Portugal. Tel.: +351 217519393; fax: +351 217526400.

E-mail addresses: elsa.dias@insa.min-saude.pt, elsa_dias@yahoo.co.uk (E. Dias).
}

senescence phase, can release high proportions of MC into water column/surface (van Apeldoorn et al., 2007). The ingestion of contaminated raw water or inefficiently treated drinking water is the principal route for human exposure to MC (Codd, 2000). Human intoxication can also occur through haemodialysis treatment if the water that supplies the haemodialysis unit is contaminated with toxic cyanobacteria. Although less frequent, this route is the most harmful due to the direct enter of microcystins into the blood stream (Codd, 2000).

The hepatotoxicity of MC is relatively well characterized both in vivo and in vitro models (revised in Duy et al., 2000; van Apeldoorn et al., 2007). The liver specificity of MC is due to their selective uptake by hepatocytes through the membrane transport family OAPT - Organic Anion Polypeptide Transporters - that mediates the uptake and elimination of numerous xenobiotics (Hagenbuch and Meier, 2003; Fischer et al., 2005). Inside the hepatocytes, MCLR inhibits the serine/threonine protein phosphatases 1 and 2A (Yoshizawa et al., 1990). The inhibition of PP1 and PP2A induces the hyperphosphorylation of cytoskeletal proteins, which leads to hepatocyte deformation, with the consequent collapse of liver tissue organization, liver necrosis, liver haemorrhage and death (Falconer and Yeung, 1992).

Based on animal sub-chronic toxicological data, the World Health Organization (WHO, 2008) has established a guideline for microcystins in drinking water ( $1 \mathrm{nM}$ of $\left.\mathrm{MCLR}_{\text {equiv }}\right)$. This guideline 
is a provisional preventive measure to human health, as the toxicological database is limited (WHO, 2008). Besides the liver, the effects of microcystins on other organs have been barely investigated.

In fact, although OATPs are absent, or less expressed, in most non-liver cells (Fischer et al., 2005) some of the specific MC carriers have been already identified in human kidney (OATPA, OATPB) and intestine (OATPB) (Hagenbuch and Meier, 2003). In addition, some studies described in vivo toxic effects of MCLR in the kidney (Nobre et al., 1999; Milutinović et al., 2002, 2003), intestine (Botha et al., 2004; Gaudin et al., 2008), brain (Maidana et al., 2006), lungs (Soares et al., 2007) and reproductive system (Ding et al., 2006).

Epidemiological data has suggested an association between human chronic exposure to low levels of microcystins in drinking water and an increase in primary hepatocellular (Yu, 1995; Ueno et al., 1996) and colorectal cancers (Zhou et al., 2002). However, confounding risk factors associated with other contaminants or organisms were not ruled out and information about the levels of exposure is very limited (IARC, 2006). Consequently, an unequivocal cause-effect relationship between human exposure to microcystins and cancer development was not yet established. Nevertheless, in vivo two-stage rodent carcinogenesis assays have demonstrated that MCLR induces tumour promotion in rat liver (NishiwakiMatsushima et al., 1992) and mouse skin (Falconer, 1991) and tumour progression in mouse colon (Humpage et al., 2000). Based on its tumour promoting activity, MCLR is presently classified by IARC (2006) as a potential carcinogen to humans (class 2B).

The mechanisms underlying the MCLR-induced tumour promotion are largely unknown. However, it was suggested that mitogen-activated protein kinases (MAPK) may be involved in the tumour promotion activity of MCLR given its ability to inhibit protein phosphatases PP1 and PP2A (Gehringer, 2004). The MAPK cascade Ras-Raf-MEK1/2-ERK1/2 has a key role in cellular proliferation and is regulated by several types of phosphatases including the serine/ threonine phosphatases PP1 and PP2A (Junttila et al., 2008). PP2A, in particular, has primarily an inhibitory effect on the pathway, namely on the Ras, MEK1/2 and ERK1/2 components (Junttila et al., 2008). Thus, it could be hypothesised that by inhibiting PP2A, MCLR deregulates the ERK1/2 pathway and promotes cell proliferation.

The involvement of the ERK1/2 pathway in MCLR-mediated tumour promotion has been supported only by few studies. Li et al. (2009) demonstrated that an extract of microcystins purified from a cyanobacterial bloom induced the activation the proto-oncogenes $c$-jun, $c$-fos e c-myc in mouse liver, kidney and testis. In fact, Fos and Jun proteins form the composite transcription factor activating protein-1 (AP-1), a mitogen-activating transactivator important for cell proliferation (Turatti et al., 2005). It is known that active ERK1/2 accumulates in the nucleus and activates transcription factors (Elk-1 and SAP 1A) that stimulate the transcription of c-Fos (Gilley et al., 2009) and that the activation of ERK1/ 2 stimulates the activity of AP- 1 complex. This induces expression of cyclin D and promotes cell cycle progression (Chang and Karin, 2001; Fang and Richardson, 2005; Meloche and Pouysségur, 2007).

Zhu et al. (2005) have demonstrated that MCLR induces the transformation of immortalized colorectal crypt cells through the constitutive activation of AKT and MAPK (p38 and JNK) cascades. This supports the hypothesis that MCLR represents a risk of colorectal cancer because cellular transformation is an initial step of carcinogenesis (Zhu et al., 2005). They also found that MCLR activates Ras and Raf, but without further effect on ERK1/2 status. Interestingly, Komatsu et al. (2007) showed that MCLR induces the phosphorylation of ERK1/2 on the embryonic kidney HEK293 cell line. However, this effect was associated with apoptosis and not with cell survival and growth.

In previous studies we demonstrated that MCLR induces cytotoxic effects (organelle changes/damages, cell lysis, apoptosis and necrosis) on the kidney-derived Vero-E6 cell line (Alverca et al., 2009; Dias et al., 2009). These effects were observed above $5 \mu \mathrm{M}$, according to a dose and time- dependent manner. The purpose of the present work was to evaluate the effects of low (non-cytotoxic) levels of MCLR $(0.5 \mathrm{nM}-5 \mu \mathrm{M})$ on the activation status of MAP kinases and on the stimulation of cell cycle progression and proliferation in the same cell line.

\section{Materials and methods}

\subsection{Microcystin-LR and cyanobacterial extracts}

Microcystin-LR was purchased from Sigma-Aldrich (CAS Number $101043-37-2$ ) as a white solid film (purity $\geqslant 95 \%$, by HPLC). A stock solution of MCLR ( $1 \mathrm{mM}$ ) was prepared by dissolving the toxin in MEM cell culture medium (see following section). This solution was sterilized by filtration through $0.22 \mu \mathrm{m}$ filters and kept at $-20^{\circ} \mathrm{C}$ until use. This form of MCLR is thereafter named "pure MCLR". Work solutions were prepared by diluting this solution in MEM culture medium.

The cyanobacteria extracts were prepared from two Microcystis aeruginosa strains (LMECYA 110 and LMECYA 127) isolated in 1996 from a natural bloom at Montargil reservoir, Portugal (Valério et al., 2009a) and successfully maintained in the laboratory as monoalgal, free of eukaryotes, non-axenic cultures. The production of microcystin by those strains was fully characterized by Valério et al. (2009b). SPE-HPLC-DAD analysis of cyanobacterial extracts was made according to International Standard ISO 20179 (2005), confirming the production of MCLR by LMECYA 110 and the LMECYA 127 inability to produce microcystins. This strain was tested as a negative control of $M$. aeruginosa matrix. Cultures of both isolates were performed in $2.5 \mathrm{~L}$ plankton light reactors (Aqua-Medic, Bissendorf, Germany) containing Z8 medium (Skulberg and Skulberg, 1990) under continuous aeration, in a $14 / 10 \mathrm{~h} \mathrm{~L} / \mathrm{D}$ cycle (light intensity $16 \pm 4 \mu \mathrm{E} \mathrm{m}^{-2} \mathrm{~s}^{-1}$, approx.) at $20 \pm 1^{\circ} \mathrm{C}$. Cells harvested during exponential growth phase were lyophilized in a freeze drier (Micromodul Y10, Savant, NY, USA) and extracted with a $75 \%$ methanol solution $(10 \mathrm{~mL}$ per $100 \mathrm{mg}$ of freeze dried material) overnight at $4{ }^{\circ} \mathrm{C}$ under magnetic stirring. The extract was further sonicated with an ultrasonic probe (Sonics Vibra-Cell CV33, Sonics \& Materials Inc., CA, USA), centrifuged and evaporated at $35^{\circ} \mathrm{C}$ (Buchi-R, Flawil, Switzerland) to eliminate the alcoholic fraction. The resulting aqueous extract was subjected to solid phase extraction for microcystin clean-up on Sep-PakC18 cartridges (500 mg, Millipore, Bedford, MA, USA) preconditioned with methanol and equilibrated with distilled water. The MCLR containing fraction was eluted with methanol $(80 \%, v / v)$ and evaporated to dryness. The solid residue was resuspended in $50 \mathrm{mM}$ acetic acid and purified by preparative chromatography through Bio-Gel P2 $(40-90 \mu \mathrm{m}$, Bio-Rad Inc., CA, USA) packed column (Amersham Biosciences, XK 26/40, i.d./ length). The mobile phase consisted of $50 \mathrm{mM}$ acetic acid and the flow rate was set at $1 \mathrm{~mL} \mathrm{~min}{ }^{-1}$ (Knauner WellChrom K-120 pumps, Germany). The elution fractions $(5 \mathrm{~mL})$ were collected on a fraction collector (Bio-Rad Mod. 2110, CA, USA) and analysed by HPLC-DAD according to the ISO standard method 20179 using commercially available MCLR standards (Alexis Biochemicals, CA, USA). MCLR-containing fractions of LMECYA 110 and correspondent fractions from LMECAY 127 were dried in a Speed-Vac system (AES 1000, Savant), re-suspended in MEM culture medium, sterilized through PVDF $0.22 \mu \mathrm{m}$ syringe filters and kept at $-20{ }^{\circ} \mathrm{C}$ until use. Those final extracts was further analysed by HPLC-DAD to confirm the MCLR concentration. Work solutions were prepared by diluting the purified extracts in MEM culture medium. 


\subsection{Vero-E6 cell line culturing}

The Vero-E6 cell line (kidney epithelial cells derived from the African green monkey - Cercopithecus aethiops) was obtained from the American Type Culture Collection (ATCC-CRL 1586). All media and supplements were purchased from Invitrogen (Paisley, UK). Cells were grown in $25 \mathrm{~cm}^{2}$ flasks (Nunc, Roskilde, Denmark) in Modified Eagle Medium (MEM) supplemented with 10\% FBS, $0.1 \mathrm{mM}$ non-essential aminoacids and $1 \mathrm{mM}$ sodium pyruvate, in a $5 \% \mathrm{CO}_{2}$ humidified incubator at $37^{\circ} \mathrm{C}$. Cells in exponential growth phase were detached from the growth surface (trypsin, $0.5 \%$ ), centrifuged $(300 \mathrm{~g})$ and the cell viability was determined by the trypan blue dye exclusion method (Philips, 1973).

\subsection{Evaluation of MCLR effect on ERK1/2 activation}

\subsubsection{Cell exposure to cyanobacterial extracts and pure MCLR}

Vero-E6 cells were seeded in $35 \mathrm{~mm}$ culture dishes and cultured for $24 \mathrm{~h}$ for cell adherence and growth. The growth medium was replaced by serial dilutions of the LMECYA 110 extract in fresh growth medium, corresponding to final concentrations of MCLR ranging from 5 to $5000 \mathrm{nM}$ of MCLR. The same biomass dilutions (from 0.56 to $556 \mu \mathrm{g} \mathrm{mL}^{-1}$ ) of the non-toxic LMECYA 127 extract were tested in parallel as a control of $M$. aeruginosa extract matrix. Serial dilutions of pure MCLR stock solution containing 5-5000 nM of toxin were also tested. The cells were exposed to LMECYA extracts and pure MCLR for $24 \mathrm{~h}$ prior to MAPK analysis by immunoblotting (ERK1/2, P38 and JNK in cells exposed to LMECYA 110; ERK1/2 in cells exposed to LMECYA 127 and pure MCLR). The negative control consisted of cells grown in fresh culture medium. The positive control of the assay consisted in cells exposed to Epidermal Growth Factor - EGF $\left(10 \mathrm{ng} \mathrm{mL} \mathrm{L}^{-1}\right.$, $5 \mathrm{~min})$ or anisomycin $(0.25 \mu \mathrm{M}, 1 \mathrm{~h})$. Experiments were performed in two ( $M$. aeruginosa extracts) or three (pure MCLR) independent experiments.

\subsubsection{Cell lysis, SDS-PAGE and Western blot analysis of ERK1/2}

After toxin exposure, cells were washed with PBS and lysed with $2 \times$ Laemmli buffer. Lysates were denaturated at $95^{\circ} \mathrm{C}$ for 10 min and proteins were separated on $10 \%$ SDS-polyacrylamide gels. After electrophoresis, proteins were transferred onto PVDF (polyvinylidene difluoride) membranes (Bio-Rad) and probed with either mouse-anti-phospho-ERK1/2, anti-phospho-JNK or antiphospho-p38 antibodies from Sigma-Aldrich (dilution 1/500, Madrid, Spain). The same membranes were stripped (200 mM NaOH, $5 \mathrm{~min}$ ) and probed with rabbit-anti-ERK1/2 antibody (dilution 1/ 1000 , Cone MAPK-YT, Sigma-Aldrich) and mouse-anti- $\alpha$-tubulin (dilution 1/1000, Sigma-Aldrich) as loading controls. Specific binding was detected after incubation with goat-anti-rabbit or goatanti-mouse peroxidase-conjugate antibodies (dilution 1/3000, Bio-Rad) by Enhanced ChemiLuminescence (ECL) detection.

\subsection{G1/S progression assay}

The effect of MCLR on cell proliferation was determined using the BrdU assay, which is based on the measurement of incorporation of 5-bromo-2-deoxyuridine during DNA synthesis. Vero-E6 cells $\left(2 \times 10^{5}\right)$ were seeded on $10 \times 10 \mathrm{~mm}$ cover slips placed in $35 \mathrm{~mm}$ culture dishes and were maintained for $24 \mathrm{~h}$ to allow cell attachment and growth. Culture medium was then replaced by toxin exposure medium supplemented with $1 \%$ of FBS and containing $0.5,1,5$ or $10 \mathrm{nM}$ of MCLR. Controls consisted of cells in culture medium supplemented with $1 \%$ or $10 \%$ of FBS. After $23 \mathrm{~h}$ of exposure, $60 \mu \mathrm{M}$ BrdU (Sigma) was added for $1 \mathrm{~h}$. Cells were washed with PBS, fixed in $4 \%(\mathrm{v} / \mathrm{v})$ paraformaldehyde in PBS for $30 \mathrm{~min}$ at $4{ }^{\circ} \mathrm{C}$, permeabilized with $100 \%$ methanol for $10 \mathrm{~min}$ at room tem- perature and then incubated with $4 \mathrm{~N} \mathrm{HCl}$ for 10 min to denature DNA. The cells were neutralized with $1 \mathrm{M}$ tris, $\mathrm{pH} 8.8$ and then washed $3 \times$ with PBS. Cells on the coverslips were incubated with the mouse-anti-BrdU primary antibody (Roche) (dilution $1 / 20$ ), followed by goat-anti-mouse secondary antibody conjugated with Texas Red (Jackson Immunoresearch Laboratories) (dilution 1/ 200). Coverslips were counterstained with Dapi $(1.25 \mu \mathrm{g} / \mathrm{mL})$, post-fixed with PFA, mounted on glass slides with Vectashield mounting medium (Vector Laboratories, Berlingame, CA, USA) and sealed with nail polish. All preparations were observed under a Leica TCS fluorescence microscope. At least 300 randomly chosen nuclei were scored for BrdU incorporation for each sample. This procedure was repeated in three independent experiments for each toxin concentration.

\subsection{Statistical analysis}

Results were expressed as mean values \pm standard deviation (mean \pm SD). Statistical analysis was performed by Student's $t$-test. Differences were considered significant when $p<0.05$.

\section{Results}

The effect of exposure to a MCLR-containing $M$. aeruginosa extract on the activation state of the canonical MAP kinases p38, JNK and ERK1/2 on Vero-E6 cells was analysed by Western-blot using phospho-specific antibodies (Fig. 1). Interestingly, while all the LMECYA 110 extract dilutions (containing 5, 50, 5000 and $5000 \mathrm{nM}$ of MCLR) induced a marked increase of phosphorylated ERK1/2 (p-ERK1/2 - Fig. 1), no considerable variation was found

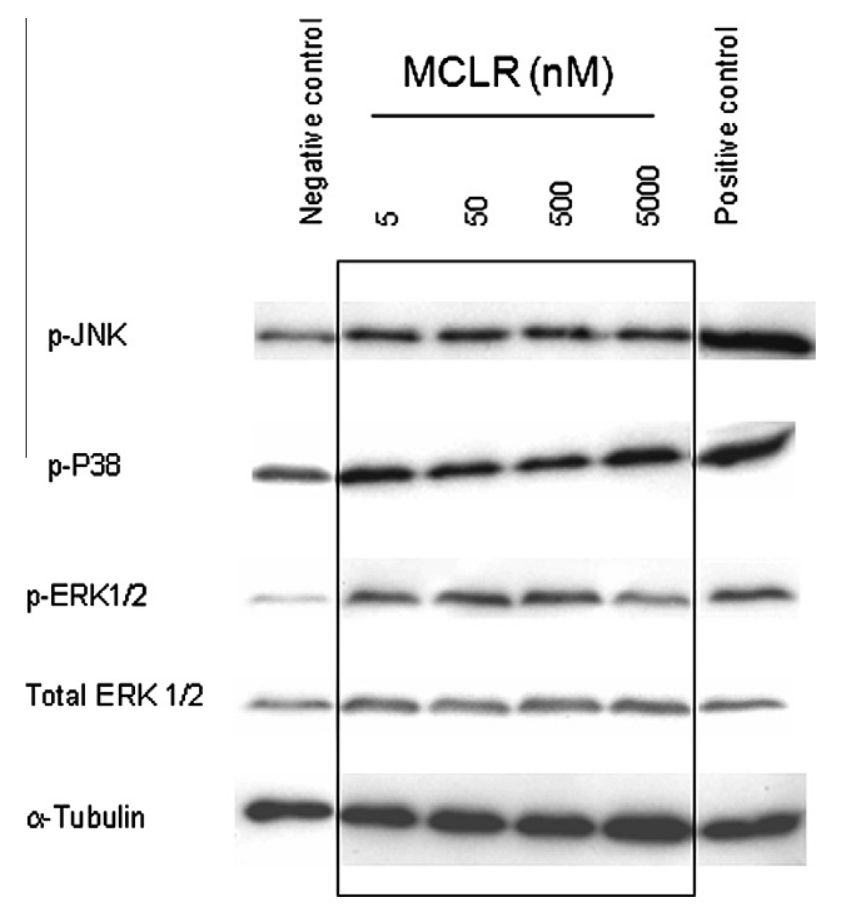

Fig. 1. Activation status of ERK1/2, p38 and JNK kinases in Vero-E6 cells by a MCLRproducer $M$. aeruginosa strain (LMECYA 110). Cells were exposed for $24 \mathrm{~h}$ to LMECYA 110 extract dilutions containing 5, 50, 500 and $5000 \mathrm{nM}$ of MCLR. The levels of active (phosphorylated) ERK $1 / 2, \mathrm{p} 38$ and JNK, total ERK $1 / 2$ and $\alpha$-tubulin (as loading control of JNK and p38) were detected by Western-blot. The negative control consisted in non-treated cells. The positive controls consisted of Epidermal Growth Factor (EGF, $10 \mathrm{ng} \mathrm{mL}^{-1}, 5 \mathrm{~min}$ ) for ERK1/2 analysis and of anisomycin $(0.25 \mu \mathrm{M}, 1 \mathrm{~h})$ for JNK and p38 analysis. Results were obtained from two independent experiments. 
in the phosphorylation state of p38 and JNK (Fig. 1). To further confirm that the observed results on ERK1/2 activity were due to MCLR exposure and not to an unknown contaminant present in the LMECYA 110 extract, the experiment was repeated using equivalent dilutions of the LMECYA 127 extract and similar concentrations of commercially available MCLR. Densitometric analysis of Western-blots from independent experiments with M. aeruginosa extracts revealed that whereas exposure to LMECYA 110 extract containing low concentrations of MCLR was sufficient to induce a 1.71-3.66-fold increase in p-ERK1/2, with maximum effect at 50 $\mathrm{nM}$, none of the dilutions of the LMECYA 127 extract produced an effect on the activation state of ERK1/2 (Fig. 2A). A significant difference ( $p=0.037$ ) was found between the dose-response curves from LMECYA 110 and LMECYA 127 extracts, witch confirms the inability of LMECYA 127 to activate ERK1/2. As shown in Fig. 2B, the densitometric analysis of Western-blots from three independent experiments revealed a slightly higher overall effect in pERK1/2 for pure MCLR (2.8-4-fold increase). Moreover, the highest

A

\section{M. aeruginosa extracts}
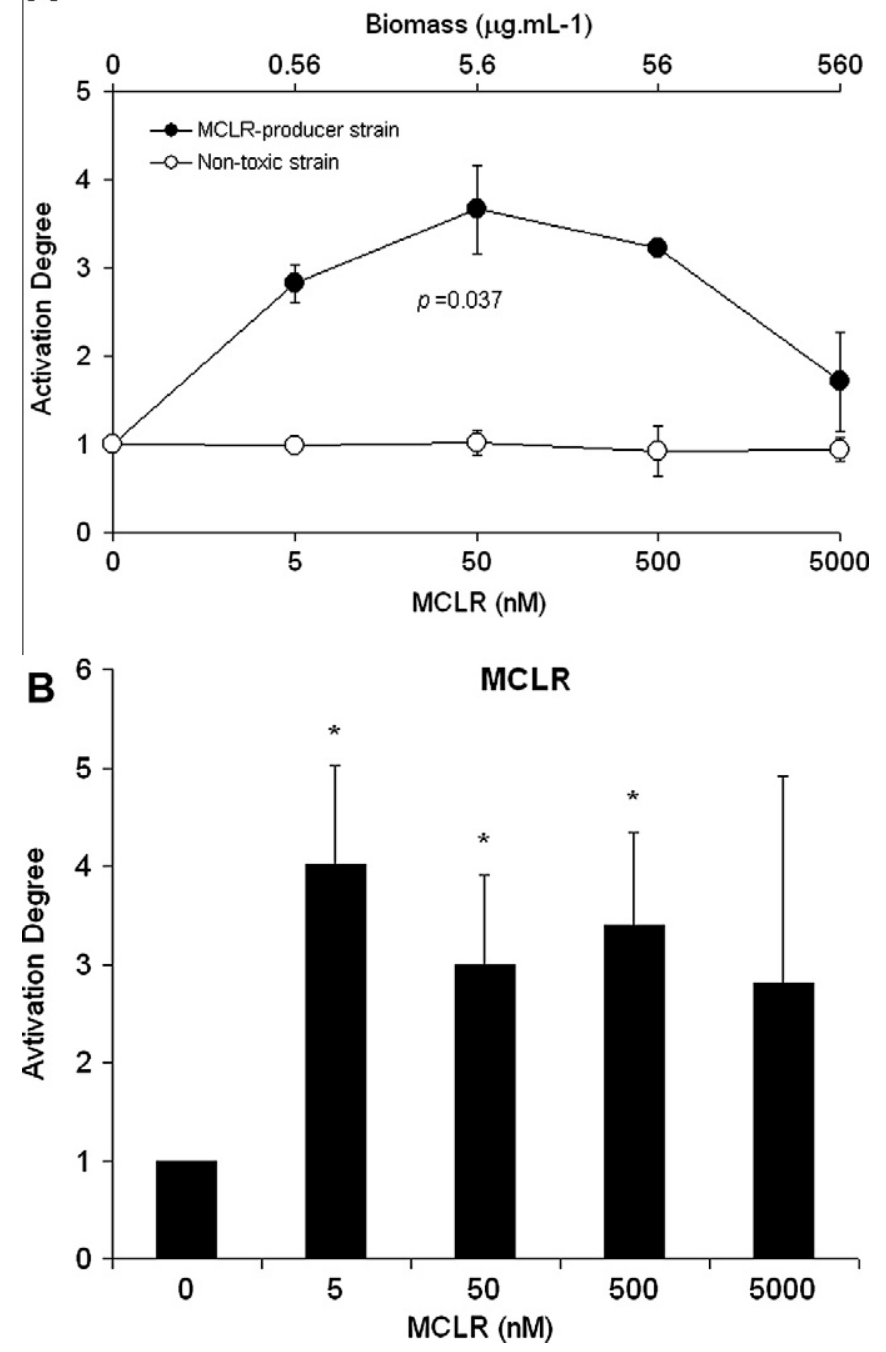

Fig. 2. Activation of ERK1/2 in Vero-E6 cells exposed for $24 \mathrm{~h}$ to (A) M. aeruginosa extracts LMECYA 110 (MCLR-producer strain) and LMECYA 127 (non-toxic strain); (B) pure MCLR. The results were obtained by densitometry analysis of Western blots and are presented as mean \pm standard deviation of treated cells in relation to negative control. In Fig. 2A, secondary $x$-axis represents the M. aeruginosa biomass concentration and $p$ indicates the significance of the difference between the two dose-response curves by a paired Student's $t$-test. In Fig. 2B, *represents a statistically significant difference between treated and control cells $(p<0.05)$. level of ERK1/2 activation was achieved at a lower toxin concentration $(5 \mathrm{nM})$ then with LMECYA 110 extract. We then chose a range of pure MCLR concentrations (0.5, 1, 5 and $10 \mathrm{nM})$ around the one with highest ERK1/2 activation ( $5 \mathrm{nM}$ ) and evaluated its effect on cell cycle progression of Vero-E6 cells by the G1/S transition BrdU assay. As shown in Fig. 3, exposure of starved cells (1\% FBS) to a range of $1-10 \mathrm{nM}$ of MCLR clearly promoted BrdU incorporation (red cells) when compared to untreated starved cells (negative control). Quantification of the observed variations revealed a statistically significant stimulation of G1/S progression at all three concentrations, with a maximum 2.2-fold increase at $5 \mathrm{nM}$ of MCLR (Fig. 4).

\section{Discussion}

In this work we demonstrated that MCLR stimulates the proliferation of kidney-derived Vero-E6 cell line in the concentration range of 1-10 nM. We showed that both pure toxin and an extract obtained from a MCLR-producer M. aeruginosa strain had no effect on the activation of p38 and JNK kinases but significantly increased ERK1/2 phosphorylation with maximum effects in the 5 and $50 \mathrm{nM}$ dose-range, respectively. This indicates that the stimulatory activity of low MCLR concentrations on Vero-E6 cell proliferation is mediated by the activation of this MAPK pathway.

MCLR is a potent inhibitor of protein phosphatases PP1 and PP2A (Yoshizawa et al., 1990). The key role of PP1 and PP2A in the regulation of cell division through the modulation of phosphorylation/dephosphorylation of signalling pathways have suggested that the MCLR-induced tumour promotion is mediated by MAPK (Gehringer, 2004).

Up to now, only few reports described the role of MAPK on MCLR-mediated toxicity. A study from Zhu et al. (2005) demonstrated that MCLR (0.1 nM) is able to transform immortalized colorectal crypt cells (NCC), rendering them anchorage independent and highly proliferative through the activation of the Akt and the p38 and JNK MAPK pathways. Since the transformation of colorectal cells is an important initial step in carcinogenesis (Zhu et al., 2005), these results support the hypothesis of MCLR being a risk factor of colorectal cancer (Zhou et al., 2002). In the same study, it was also demonstrated that members of the Ras and Raf families were activated, but without further activation of ERK1/2 kinases (Zhu et al., 2005). Here we found in Vero-E6 cells that low MCLR concentration affected the ERK1/2 but not the p38 and JNK MAPK pathways. The non-activation of P38 and JNK should be further confirmed by testing more exposure conditions to MCLR (time of exposure and toxin concentration). Interestingly, Komatsu et al. (2007) also reported that MCLR induced the phosphorylation of ERK1/2 in human embryonic kidney (HEK) 293 cells. They described that the activation of ERK1/2 occurred at the same toxin concentration ( $50 \mathrm{nM}$ ) that induced apoptosis and concluded that, in this cell line, apoptosis is mediated by ERK1/2. However, the HEK293 cells studied were transfected with the human hepatocyte uptake transporters OATP1B1 and OATP1B3. Therefore, the toxicokinetics of MCLR in transfected HEK293 cells is surely quite distinct from non-OATP transfected cells, given the fact that OATP expression in immortalized cell lines is reduced or even abolished (Boaru et al., 2006). Hence, the putative higher toxin absorption and accumulation in these HEK293 cells might justify their higher sensitivity to MCLR compared with Vero-E6 cells, which presented cytotoxic effects only for MCLR concentrations in the range of $\mu \mathrm{M}$ (Dias et al., 2009). Moreover, our study revealed that ERK1/2 is activated in Vero-E6 cells within a broad range of concentrations $(5 \mathrm{nM}-5 \mu \mathrm{M})$ whereas cell proliferation was stimulated within the range of 1-10 nM. As previously demonstrated, MCLR induces autophagy in Vero-E6 cells exposed to $5 \mu \mathrm{M}$, followed by apoptosis 


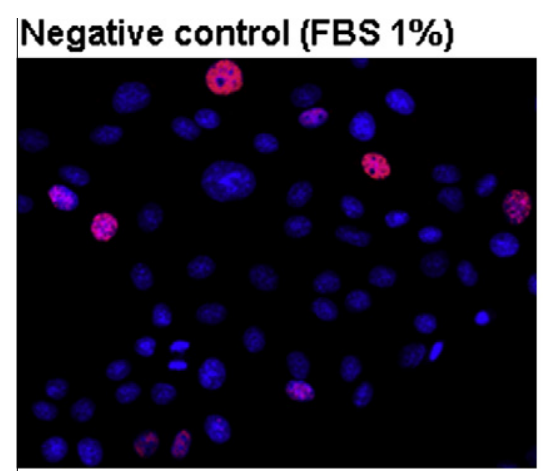

MCLR $0.5 \mathrm{nM}$

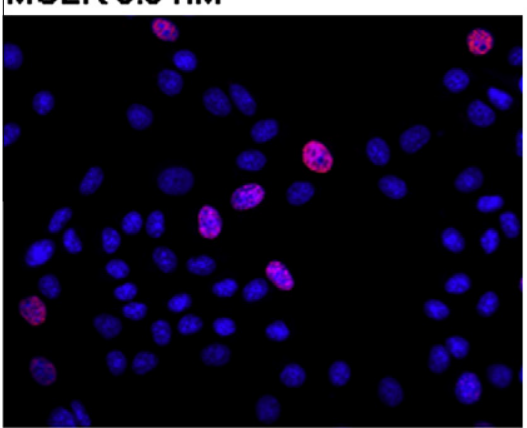

MCLR 5 nM

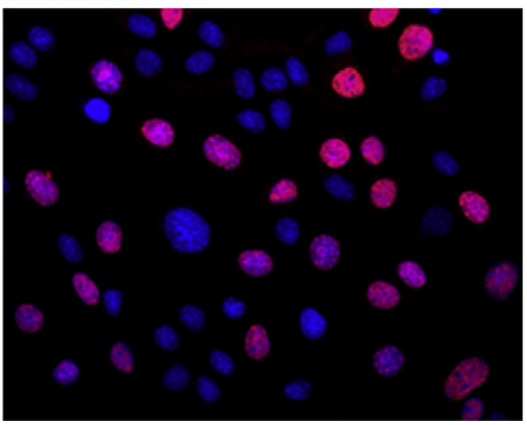

Positive control (FBS 10\%)

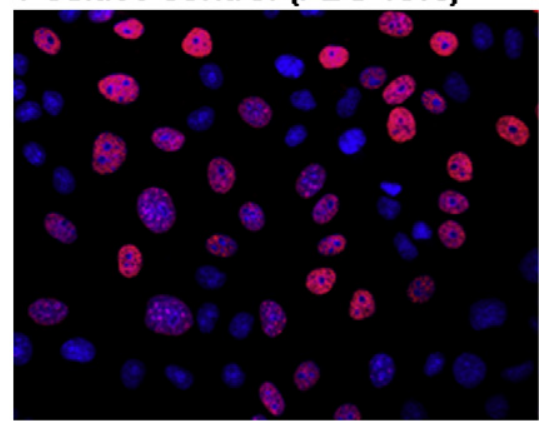

MCLR $1 \mathrm{nM}$

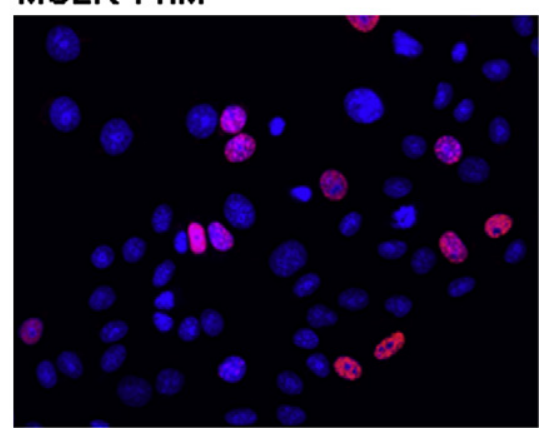

MCLR $10 \mathrm{nM}$

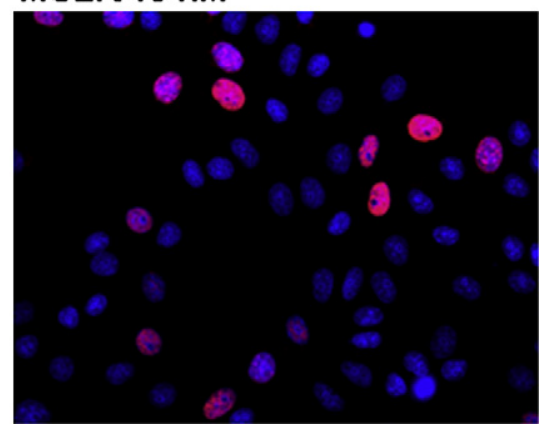

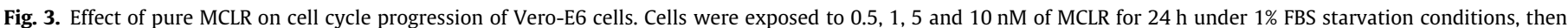

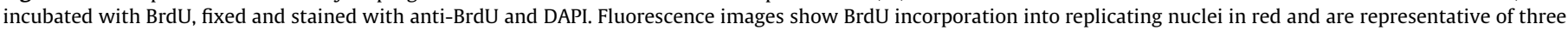

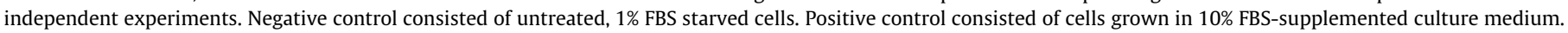
(For interpretation of the references to colour in this figure legend, the reader is referred to the web version of this article.)

above $20 \mu \mathrm{M}$ (Alverca et al., 2009). Autophagy is a process that eliminates damaged or redundant organelles and misfolded proteins, contributing to the maintenance of cell homeostasis and survival. However, it could also function as a cell death mechanism that might collaborate with apoptotic cell death (Codogno and Meijer, 2005; Eskelinen and Saftig, 2009). Notably, the ERK1/2 pathway has been implicated in the positive regulation of autophagy (Ogier-Nenis et al., 2000; Pattingre et al., 2003; Meijer and Codogno, 2004) and in the inhibition of apoptosis (Junttila et al., 2008). Thus, we hypothesise that the MCLR-mediated ERK1/2 activation has a binary effect in Vero-E6 cells: the stimulation of cell proliferation at low MCLR concentrations and the induction of autophagic (pre-apoptotic) mechanisms at (sub)cytotoxic concentrations of MCLR. The role of ERK1/2 activation on the MCLR-mediated autophagic response as well as its eventual contribution to the inhibition of apoptosis would significantly improve the knowledge of the mechanisms underlying MCLR tumour promoting activity.

Although the main target organ of MCLR is the liver and despite most of the toxin being excreted via biliar route, a small (9\%) per- centage of the toxin (free or conjugated with GSH and Cys) is also eliminated through the urine (Robinson et al., 1990; Ito et al., 2002). It should be noted that although MCLR metabolites are less toxic than non-metabolized toxin, they still maintain the inhibitory activity of protein phosphatases PP1 and PP2A (Ito et al., 2002). In this way, the nephrotoxic effects of MCLR should not be disregarded, in particular those related with chronic exposure to low levels of toxin.

In conclusion, this work demonstrates that MCLR (pure or extracted from cyanobacteria) stimulates the G1/S transition of the kidney cell line Vero-E6 at very low concentrations (circa 5000 times lower than those that induce cytotoxic effects). The degree of ERK1/2 activation and subsequent G1/S cell cycle progression that was observed following MCLR treatment is comparable to that described for other tumorigenic stimuli (Moniz et al., 2007; Moniz and Peter, 2010) and thus biologically meaningful. Considering that the ERK1/2 pathway is known to be upregulated in approximately one-third of all human cancers (Dhillon et al., 2007), our results emphasise the importance to confirm in vivo the impact of MCLR on tumour promotion at kidney level. 


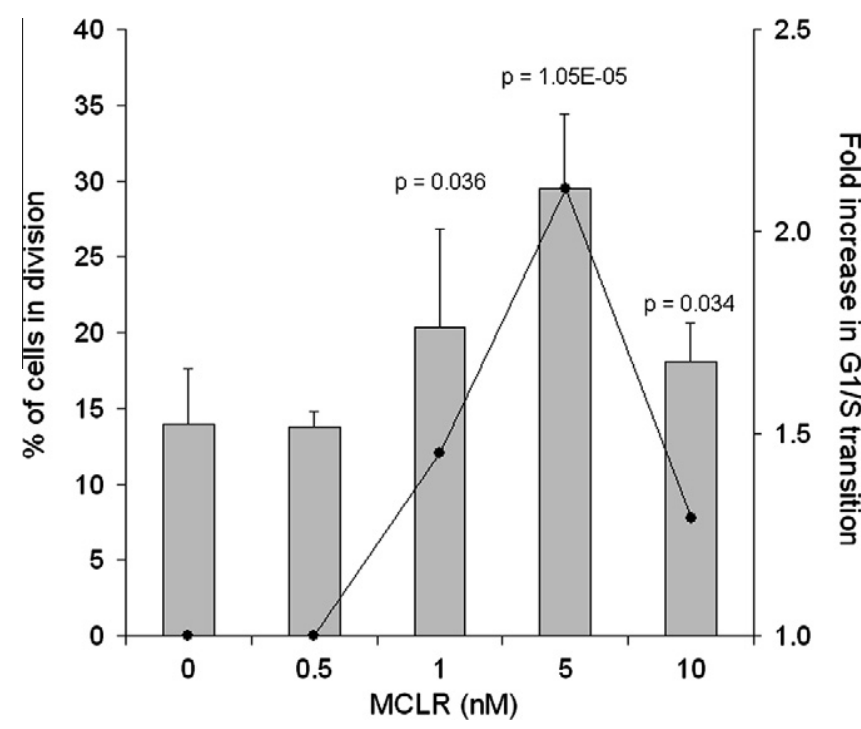

Fig. 4. Quantification of the effect of pure $\operatorname{MCLR}(0.5,1,5$ and $10 \mathrm{nM})$ on Vero-E6 cell proliferation. BrdU incorporation was scored in at least 300 randomly chosen nuclei per sample and repeated in three independent experiments.Bars represent the mean percentage of replicating cells ( \pm standard deviation). Line represents the induced fold increase in G1/S transition, in relation to the negative control cells. Results were obtained from three independent experiments. $p$ indicates the significance of the difference between treated and control cells.

\section{Conflict of interest statement}

The authors declare that there are no conflicts of interest.

\section{Acknowledgements}

This work was supported by the Portuguese Foundation for Science and Technology via Grant SFRH/BD/10585/2002 attributed to Elsa Dias and pluriannual programs for the Centre for Research on Human Molecular Genetics (CIGMH) and Institute for Medicine and Pharmaceutical Sciences (I-MED).

\section{References}

Alverca, E., Andrade, M., Dias, E., Sam-Bento, F., Batoéru, M.C.C., Jordan, P., Silva, M.J., Pereira, P., 2009. Morphological and ultrastructural effects of microcystin-LR from Microcystis aeruginosa extract on a kidney cell line. Toxicon 54, 283-294.

Boaru, D.A., Dragoş, N., Schirmer, K., 2006. Microcystin-LR induced cellular effects in mammalian and fish primary hepatocyte cultures and cell lines: a comparative study. Toxicology 218, 134-148.

Botha, N., Van de Venter, M., Downing, T.G., Shepard, E.G., Gehringer, E.G., 2004. The effect of intraperitoneally administered microcystin-LR on the gastrointestinal tract of Balb/c mice. Toxicon 43, 251-254.

Chang, L., Karin, M., 2001. Mammalian MAP kinase signalling cascades. Nature 410, 37-40.

Chen, Y.M., Lee, T.H., Lee, S.J., Huang, H.B., Huang, R., Chou, H.N., 2006. Comparison of protein phosphatase inhibition activities and mouse toxicities of microcystins. Toxicon 47, 742-746.

Codd, G.A., 2000. Cyanobacterial toxins, the perception of water quality, and the prioritisation of eutrophication control. Ecol. Eng. 16, 51-60.

Codd, G.A., Morrison, L.F., Metcalf, J.S., 2005. Cyanobacterial toxins. Risk management for health protection. Toxicol. Appl. Pharmacol. 203, 264-272.

Codogno, P., Meijer, A.J., 2005. Autophagy and signaling: their role in cell survival and death. Cell Death Different. 12, 1509-1518.

Dhillon, A.S., Hagan, S., Rath, O., Kolch, W., 2007. MAP kinase signalling pathways in cancer. Oncogene 26, 3279-3290.

Dias, E., Andrade, M., Alverca, E., Pereira, P., Batoréu, M.C.C., Jordan, P., Silva, M.J., 2009. Comparative study of the cytotoxic effect of microcystin-LR and purified extracts from Microcystis aeruginosa on a kidney cell line. Toxicon 53, 487-495.

Ding, X.S., Li, X.Y., Duan, H.Y., Chung, I.K., Lee, J.A., 2006. Toxic effects of microcystis cell extracts on the reproductive system of male mice. Toxicon 48, 973-979.

Duy, T.N., Lam, P.K.S., Shaw, G.R., Connell, D.W., 2000. Toxicology and risk assessment of freshwater cyanobacterial (blue-green algae) toxins in water. Rev. Environ. Contam. Toxicol. 163, 113-186.
Eskelinen, E.L., Saftig, P., 2009. Autophagy: a lysosomal degradation pathway with a central role in health and disease. Biochem. Biophys. Acta 1793, 664-673.

Falconer, I.R., 1991. Tumour promotion and liver injury caused by oral consumption of cyanobacteria. Environ. Toxicol. Water Qual. 6, 177-184.

Falconer, I.R., Yeung, D.S., 1992. Cytoskeletal changes in hepatocytes induced by microcystis toxins and their relation to hyperphosphorylation of cell proteins. Chem. Biol. Interact. 81, 181-196.

Fang, J.L., Richardson, B.C., 2005. The MAPK signalling pathways and colorectal cancer. Lancet Oncol. 6, 322-327.

Fischer, W.L., Altheimer, S., Cattori, V., Meier, P.J., Dietrich, D.R., Hagenbuch, B. 2005. Organic anion transporting polypeptides expressed in liver and brain mediate uptake of microcystin. Toxicol. Appl. Pharmacol. 203, 257-263.

Funari, E., Testai, E., 2008. Human health risk assessment related to cyanotoxin exposure. Crit. Rev. Toxicol. 38, 97-125.

Gaudin, J., Huet, S., Jarry, G., Fessard, V., 2008. In vivo DNA damage induced by the cyanotoxin microcystin-LR: comparison of intra-peritoneal and oral administration by use of the comet assay. Mutat. Res. 652, 65-71.

Gehringer, M.M., 2004. Microcystin-LR and okadaic acid-induced cellular effects: a dualistic response. FEBS Lett. 557, 1-8.

Gilley, R., March, H.N., Cook, S.J., 2009. ERK1/2, but not ERK5, is necessary and sufficient for phosphorylation and activation of c-Fos. Cell. Signal. 21, 969-977.

Hagenbuch, B., Meier, P.J., 2003. The superfamily of organic anion transporting polypeptides. Biochim. Biophys. Acta 1609, 1-18.

Humpage, A.R., Hardy, S.J., Moore, E.J., Froscio, S.M., Falconer, I.R., 2000. Microcystins (cyanobacterial toxins) in drinking water enhance the growth of aberrant crypt foci in the mouse colon. J. Toxicol. Environ. Health A 61, 155-165.

IARC, 2006. Cyanobacterial peptide toxins. Available from: <http://monographs. iarc.fr/ENG/Meetings/94-cyanobacteria.pdf>.

ISO 20179, 2005. Water quality - determination of microcystins - method using solid phase extraction (SPE) and high performance liquid chromatography (HPLC) with ultraviolet (UV) detection.

Ito, E., Takai, A., Kondo, F., Masui, H., Imanishi, S., Harada, K.I., 2002. Comparison of protein phosphatase inhibitory activity and apparent toxicity of microcystins and related compounds. Toxicon 40, 1017-1025.

Junttila, M.R., Li, S.P., Westermarck, J., 2008. Phosphatase-mediated crosstalk between MAPK signalling pathways in the regulation of cell survival. FASEB J. 22, 954-965.

Komatsu, M., Furukawa, T., Ikeda, R., Takumi, S., Nong, Q., Aoyama, K., Akiyama, S.I., Keppler, D., Takeuchi, T., 2007. Involvement of mitogen-activated protein kinase signalling pathways in microcystins-LR-induced apoptosis after its selective uptake mediated by OATP1B1 and OATP1B3. Toxicol. Sci. 97, 407-416.

Li, H., Xie, P., Li, G., Hao, L., Xiong, Q., 2009. In vivo study on the effects of microcystin extracts on the expression profiles of proto-oncogenes (c-fos, c-jun and c-myc) in liver, kidney and testis of male Wistar rats injected i.v. with toxins. Toxicon 53, 169-175.

Maidana, M., Carlis, V., Galhardi, F.G., Yunes, J.S., Geracitano, L.A., Monserrat, J.M., Barros, D.M., 2006. Effects of microcystins over short- and long-term memory and oxidative stress generation in hippocampus of rats. Chem.-Biol. Interact. $159,223-234$.

Meijer, A.J., Codogno, P., 2004. Regulation and role of autophagy in mammalian cells. Int. J. Biochem. Cell Biol. 36, 2445-2462.

Meloche, S., Pouysségur, J., 2007. The ERK1/2 mitogen-activated protein kinase pathway as a master regulator of the G1- to S-phase transition. Oncogene 26 3227-3239.

Milutinović, A., Sedmark, B., Horvat-ŽnidarŠić, I., Šuput, D., 2002. Renal injuries induced by chronic intoxication with microcystins. Cell. Mol. Biol. Lett. 7, 139141.

Milutinović, A., Živin, M., Zorc-Plesković, R., Sedmark, B., Šuput, D., 2003. Nephrotoxic effects of chronic administration of microcystins-LR and -YR. Toxicon 42, 281-288.

Moniz, S., Peter, P., 2010. Emerging roles for WNK kinases in cancer. Cell. Mol. Life Sci. 67, 1265-1276.

Moniz, S., Veríssimo, F., Matos, P., Brazão, R., Silva, E., Kotevelets, L., Chastre, E. Gespach, C., Jordan, P., 2007. Protein kinase WNK2 inhibits cell proliferation by negatively modulating the activation of MEK1/ERK1/2. Oncogene 26, 60716081.

Nishiwaki-Matsushima, R., Ohta, T., Nishiwaki, S., Suganuma, M., Kohyama, K., Ishikawa, T., Carmichael, W.W., Fujiki, H., 1992. Liver tumor promotion by the cyanobacterial cyclic peptide toxin microcystin-LR. J. Cancer Res. Clin. Oncol. $118,420-424$.

Nobre, A.C.L., Jorge, M.C.M., Menezes, D.B., Fonteles, M.C., Monteiro, H.S.A., 1999 Effects of microcystin-LR in isolated perfused rat kidney. Brazil. J. Med. Biol. Res. 32, 985-988.

Ogier-Nenis, E., Pattingre, S., Benna, J.E., Codogno, P., 2000. Erk1/2-dependent phosphorylation of $\mathrm{G} \alpha$-interacting protein stimulates its GTPase accelerating activity and autophagy in human colon cancer cells. J. Biol. Chem. 275, 3909039095.

Pattingre, S., Bauvy, C., Codogno, P., 2003. Amino acids interfere with the Erk1/2dependent control of macroautophagy by controlling the activation of Raf- 1 in human colon cancer HT-29 cells. J. Biol. Chem. 278, 16667-16674.

Philips, H.J., 1973. Dye exclusion tests for cell viability. In: Kruse, P.F., Patterson, M.K. (Eds.), Tissue Culture: Methods and Applications. Academic Press, NY, pp. 406-408.

Robinson, N.A., Pace, J.G., Matson, C.F., Miura, G.A., Lawrence, W.B., 1990. Tissue distribution, excretion and hepatic biotransformation of microcystin-LR in mice. J. Pharmacol. Exp. Ther. 256, 176-182. 
Soares, M., Cagido, V.R., Ferraro, R.B., Meyer-Fernandes, J.R., Rocco, P.R.M., Zin, W.A Azevedo, S.M.F.O., 2007. Effects of microcystin-LR on mouse lungs. Toxicon 50, 330-338.

Turatti, E., Neves, A.C., Magalhães, M.H.C.G., Sousa, S.O.M., 2005. Assessment of cJun, c-Fos and cyclin D1 in premalignant and malignant oral lesions. J. Oral Sci. 47, 71-76.

Ueno, Y., Nagata, S., Tsutsumi, T., Hasegawa, A., Watanabe, M.F., Park, H.D., Chen G.C., Chen, G., Yu, S.Z., 1996. Detection of microcystins, a blue-green algal hepatotoxins, in drinking water sampled in Haimen and Fusui, endemic areas of primary liver cancer in China, by highly sensitive immunoassay. Carcinogenesis 17, 1317-1321.

Valério, E, Chambel, L, Faria, N., Paulino, S., Pereira, P., Tenreiro, R., 2009a. Molecular identification, typing and traceability of cyanobacteria from freshwater reservoirs. Microbiology 155, 642-656.

Valério, E. et al., 2009b. Multiplex PCR for detection of microcystins-producing cyanobacteria from freshwater samples. Environ. Toxicol.. doi:10.1002/ Tox.20502.
Van Apeldoorn, M.E., van Egmond, H.P., Speijers, G.J.A., Bakker, G.J.I., 2007. Toxins of cyanobacteria. Mol. Nutr. Food Res. 51, 7-60.

WHO, 2008. Guidelines for Drinking Water Quality, third ed. Incorporating the First and Second Addenda, vol. 1, Recommendations. World Health Organization, Geneva.

Yoshizawa, I., Matsushima, R., Watanabe, M.F., Harada, H., Ichihara, A., Carmichael, W.W., Fujiki, H., 1990. Inhibition of protein phosphatases by microcystis and nodularin associated with hepatotoxicity. J. Cancer Res. Clin. Oncol. 116, 609614.

Yu, S.Z., 1995. Primary prevention of hepatocellular carcinoma. J. Gastroenterol. Hepatol. 10, 674-682.

Zhou, L., Yu, H., Chen, K., 2002. Relationship between microcystin in drinking water and colorectal cancer. Biomed. Environ. Sci. 15, 166-171.

Zhu, Y., Zhong, X., Zheng, S., Ge, Z., Du, Q., Zhang, S., 2005. Transformation of immortalized colorectal crypt cells by microcystin involving constitutive activation of Akt and MAPK cascade. Carcinogenesis 26, 1207-1214. 